\title{
Supporting Information for \\ Influence of Ligand Structure on Excited State Surface Chemistry of Lead Sulfide Quantum Dots
}

Eric R. Kennehan, ${ }^{1,2 \ddagger}$ Kyle T. Munson, ${ }^{3 \ddagger}$ Christopher Grieco ${ }^{1,2}$, Grayson S. Doucette, ${ }^{4}$ Ashley R. Marshall, ${ }^{5,6}$ Matthew C. Beard, ${ }^{5,6}$ John B. Asbury ${ }^{2,3,4 *}$

1. Magnitude Instruments, State College, PA 16803, USA

2. Department of Chemistry, The Pennsylvania State University, University Park, PA 16802, USA.

3. Materials Research Institute, The Pennsylvania State University, University Park, PA 16802, USA.

4. Intercollege Materials Science and Engineering Program, The Pennsylvania State University, University Park, PA 16802, USA.

5. Chemical and Materials Science, National Renewable Energy Laboratory (NREL), Golden, CO 80401, USA.

6. Department of Chemistry and Biochemistry, University of Colorado, Boulder, CO 80309, USA.

${ }^{\ddagger}$ Denotes equal contribution.

\section{Table of Contents:}

S1. Characterization of QD size and first excitonic transitions

S2. FTIR spectra of OA and I/MPA passivated PbS QD films

S3. Broadband TA spectroscopy and kinetics of oleate and I/MPA passivated PbS films

S4. References 


\section{Section S1: Characterization of QD size and first excitonic transitions}

We first characterized the QD films examined in this study using visible to near infrared absorption spectroscopy. Figure S1A shows the absorption spectra of four sizes of oleate passivated PbS QDs in octane solution that were used to deposit the nanocrystal films examined in this work. The spectra are offset for clarity. All absorption spectra were collected using a homebuilt instrument previously described. ${ }^{1}$ The spectra of the $\mathrm{PbS}$ solutions exhibit clear first excitonic transitions characteristic of quantum confined nanoparticles. ${ }^{2}$

To determine the approximate size of the PbS QDs examined here, we fit the absorption spectra appearing in Figure S1A with log-normal distributions. This analysis allowed us to compare our results to sizing curves calculated using the $\mathrm{K} \cdot \mathrm{P}$ four-band envelope method..$^{2-3} \mathrm{We}$ used the center frequency of the log-normal distributions to determine the representative QD sizes for the samples examined here. From this analysis, we found the sizes of our PbS QDs to be $2.9,3.1,3.7$, and $4.9 \pm .1 \mathrm{~nm}$, respectively.

PbS QD sizes also were characterized using transmission electron microscopy (TEM). For TEM measurements, colloidal PbS solutions were drop-cast onto carbon grids. All TEM images were collected using a FEI, Talos F200X equipped with a XFEG source at $200 \mathrm{kV}$. Figure S1B shows the TEM images of the PbS QDs. From the images, the diameters of the QDs were found to be $2.9 \pm 0.3 \mathrm{~nm}, 3.1 \pm 0.3 \mathrm{~nm}, 3.7 \pm 0.4 \mathrm{~nm}$, and $4.9 \pm 0.4 \mathrm{~nm}$, respectively. 

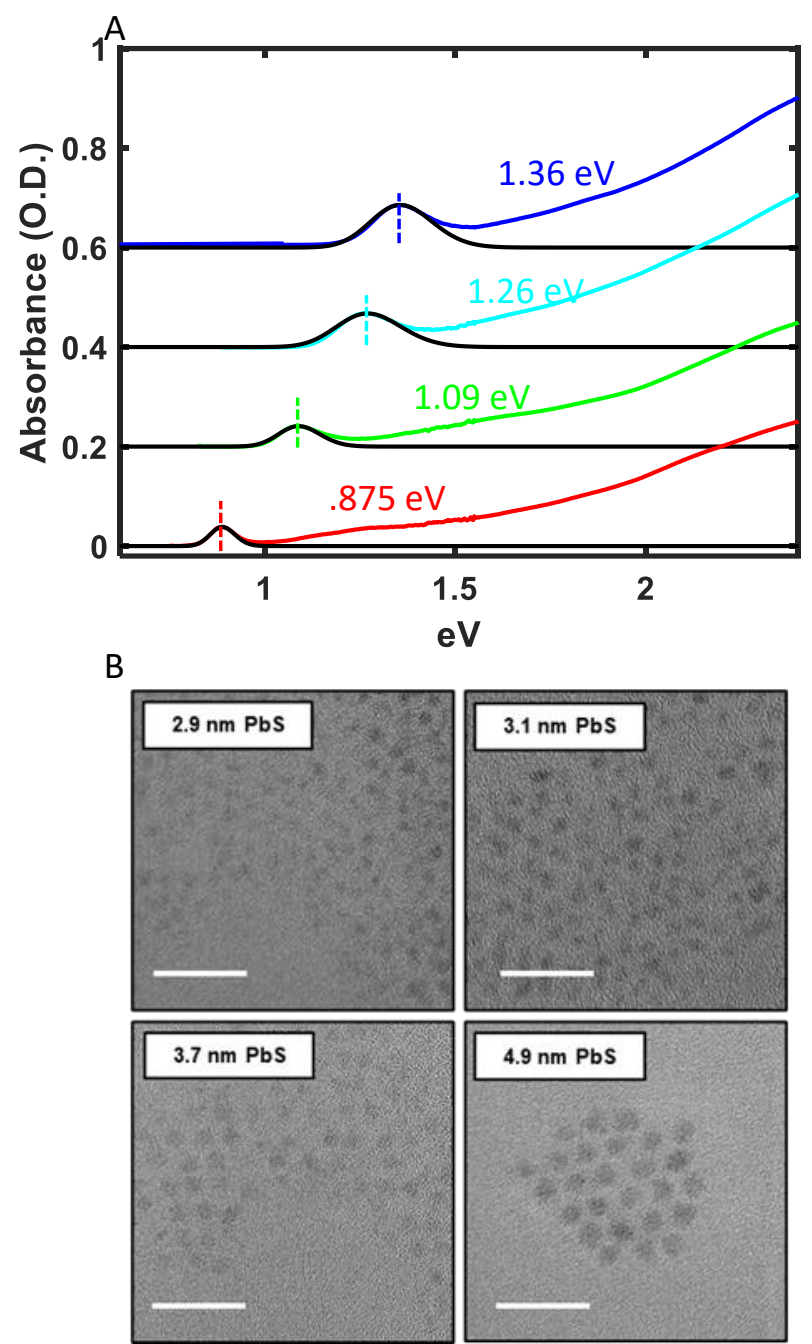

Figure S1. (A) Visible to near infrared absorption spectra of oleate passivated PbS QDs in solution. Log-normal fits to each absorption spectrum are also shown (solid black lines). The spectra are offset for clarity (B) TEM images of 2.9, 3.1, 3.7 , and $4.9 \mathrm{~nm}$ PbS quantum dots. The white scale bar is 20 $\mathrm{nm}$ for all images. 


\section{Section S2: FTIR spectra of OA and I/MPA passivated PbS QD films}

Distinct correlations have been observed between vibrational frequencies of the symmetric $\nu_{\text {sym }}$ and antisymmetric $v_{\text {as }}$ modes of carboxylate groups and their bonding geometries with metal ions. ${ }^{4-5}$ These correlations are derived from systematic measurements of mid-infrared absorption spectra of ionic compounds containing carboxylates and the corresponding crystal structures of the compounds from X-ray diffraction. Figure $\mathbf{S 2}$ depicts results of these frequency-structure correlations that are salient to the bonding of OA and MPA ligands to PbS QD surfaces. In particular, the bridging, chelating, and monodentate bonding geometries are distinguished by the difference in frequency between their $v_{\text {sym }}$ and $v_{\text {as }}$ modes as well as by the particular frequencies of these modes. For example, carboxylate groups bonded to metal ions in the chelating geometry have the smallest difference in frequency between the $v_{\text {sym }}$ and $v_{\text {as }}$ modes and have the largest metal-oxygen coordination (two oxygen atoms/metal ion). Carboxylates that bond via the bridging geometry to two metal ions exhibit a larger frequency difference and have a lower metal-oxygen coordination (one oxygen atom/metal ion). Carboxylates that bond to metal ions via the

(A)

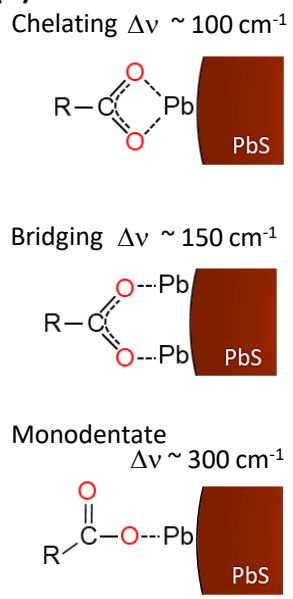

(B)

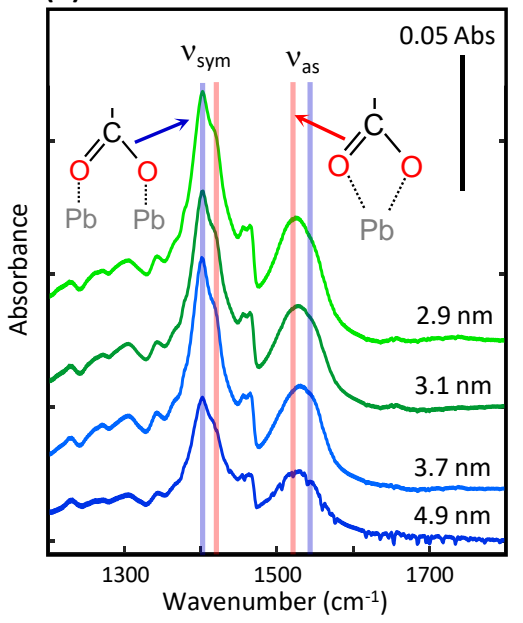

Figure S2. (A) Illustrations of common carboxylate binding motifs and the corresponding frequency differences of their carboxylate stretch modes. (B) FTIR spectra of four different size PbS QD films with oleate ligands. Distinct vibrational modes including symmetric $v_{\text {sym }}$ and antisymmetric $v_{\text {as }}$ carboxylate stretches are labeled with corresponding structures. Adapted with permission from Ref 5. Copyright 2020 American Chemical Society. 
monodentate geometry possess a formal double bonded $\mathrm{C}=\mathrm{O}$ moiety with higher vibrational frequency and a formal single bonded $\mathrm{C}-\mathrm{O}$ group with lower frequency.

It is useful to examine the vibrational spectra of carboxylate groups in their protonated states for comparison to the vibrational spectra described above to further understand the surface chemistry of the PbS QDs examined in this work. Figure S3 represents an FTIR absorption spectrum of a neat MPA liquid film sandwiched between two CaF2 optical flats. The spectra exhibit the $\mathrm{O}-\mathrm{H}, \mathrm{C}-\mathrm{H}$, and $\mathrm{S}-\mathrm{H}$ stretch modes that are expected from the protonated carboxyl, propyl chain and thiol ${ }^{6}$ functional groups in the $2500-3500 \mathrm{~cm}^{-1}$ range. Importantly, the formal $\mathrm{C}=\mathrm{O}$ stretch mode of the carboxylic acid group appears around $1700 \mathrm{~cm}^{-1}$, which is clearly distinct from the frequency around $1600 \mathrm{~cm}^{-1}$ of the $\mathrm{C}=\mathrm{O}$ group of carboxylate groups involved in the monodentate bonding geometry to metal ions. Consequently, the vibrational spectra of the carboxylate group provide clear indications when they are bonded to metal ions and what the geometry of that bonding is versus when the carboxylates are protonated and detached from metal ions. This correlation is used in the work discussed here to examine changes in the surface chemistry of PbS QDs passivated with OA and MPA ligands in their excited states.

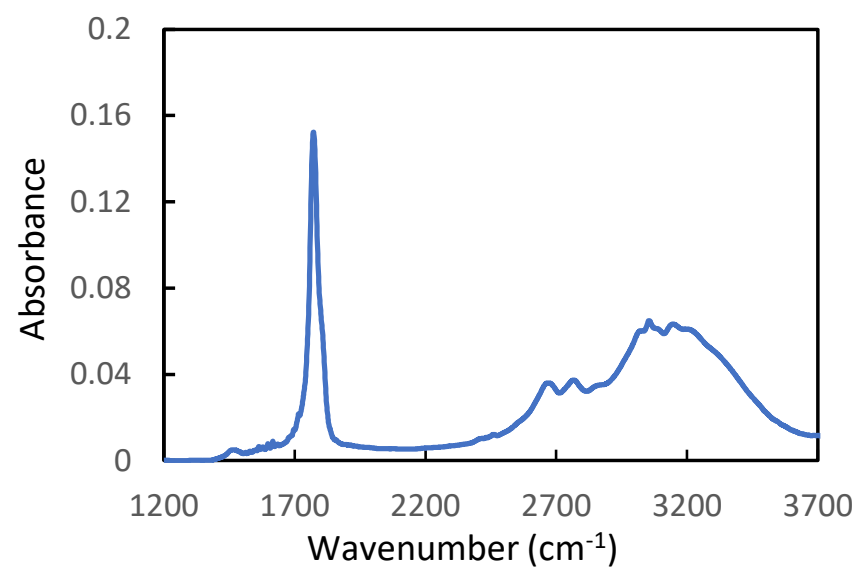

Figure S3. FTIR spectrum of a neat MPA liquid film sandwiched between two $\mathrm{CaF}_{2}$ optical flats. The formal $\mathrm{C}=\mathrm{O}$ stretch of the protonated carboxyl group at $1700 \mathrm{~cm}^{-1}$ is clearly distinct from the frequency of the formal $\mathrm{C}=\mathrm{O}$ group of carboxylate groups involved in the monodentate bonding geometry, which appears around $1600 \mathrm{~cm}^{-1}$. 


\section{Section S3: Broadband mid-IR TA spectroscopy and kinetics of oleate and I/MPA capped}

\section{PbS films}

Figure $\mathbf{S} 4$ shows the broadband mid-infrared transient absorption spectra of 2.9, 3.1, 3.7, and 4.9 nm PbS QDs capped with either oleate or I-MPA ligands measured at several time delays following $532 \mathrm{~nm}$ excitation. Figure S4A displays broadband transient absorption spectra measured in oleate passivated PbS QD films, while Figure S4B depicts the transient absorption spectra of I-/MPA passivated films. The frequencies at which the GSB and 1S-1P transitions were measured to obtain the kinetics traces in Figure $\mathbf{4}$ of the main text are marked with vertical lines for reference.
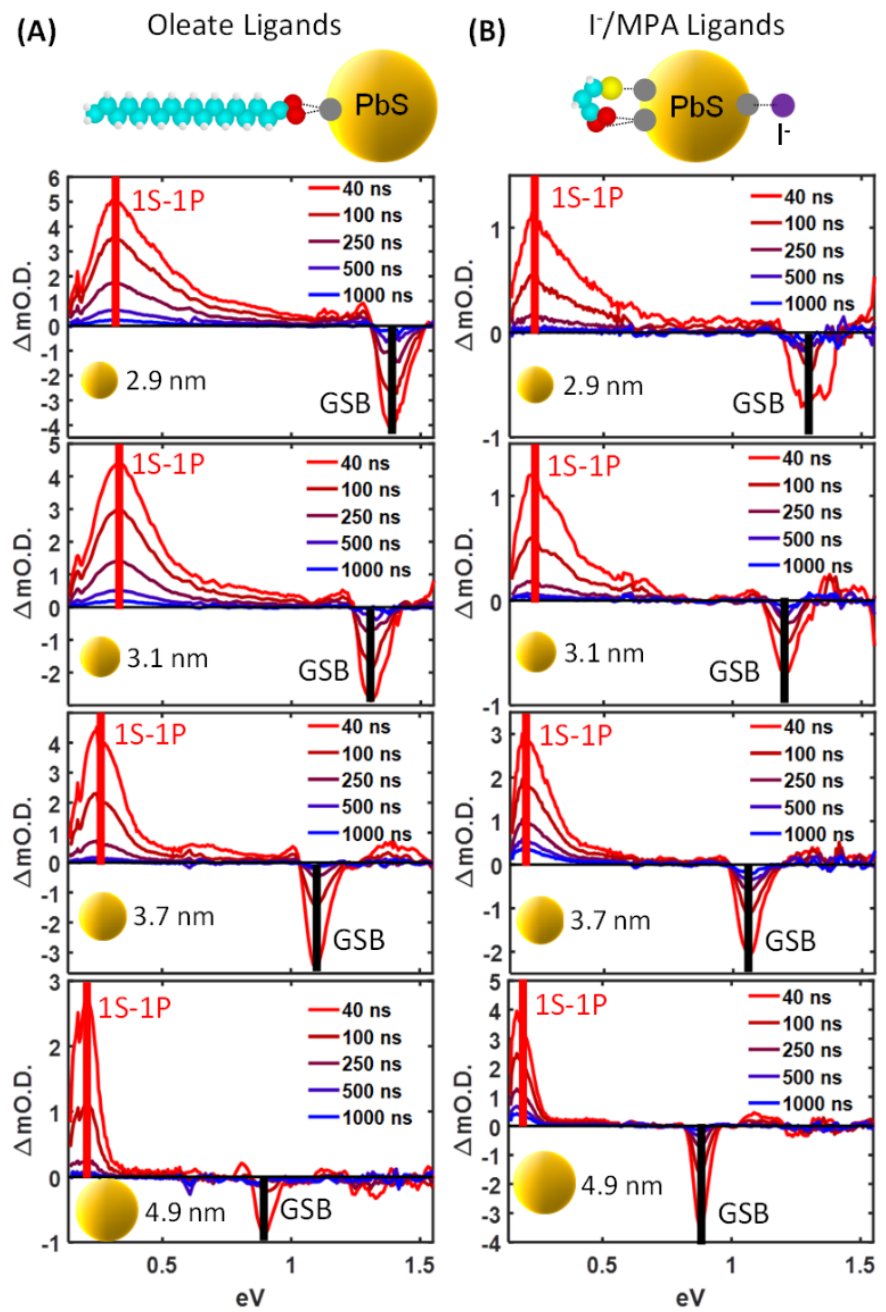

Figure S4. (A) Transient absorption spectra of 2.9, 3.1, 3.7, and $4.9 \mathrm{~nm}$ oleate passivated PbS QD films covering mid-IR to visible region with 1 S-1P and GSB transition indicated following $532 \mathrm{~nm}$ excitation. (B) Transient absorption spectra of 2.9, 3.1, 3.7, and $4.9 \mathrm{~nm}$ iodide/MPA passivated PbS QD films with 1S-1P and GSB transition indicated following $532 \mathrm{~nm}$ excitation. 
Figure S5 and Figure S6 compare the kinetics traces appearing in Figure 4 with their corresponding best fit functions for reference. Figure $\mathbf{S} 5$ depicts the kinetics traces and fits corresponding to Figure 4B for OA-PbS QD films. Figure $\mathbf{S 6}$ represents the kinetics traces and best fits for the I-/MPA-PbS QD films in Figure 4C. The best fit functions are not included in Figure 4 because their smooth curves cannot be easily distinguished from the experimental data.
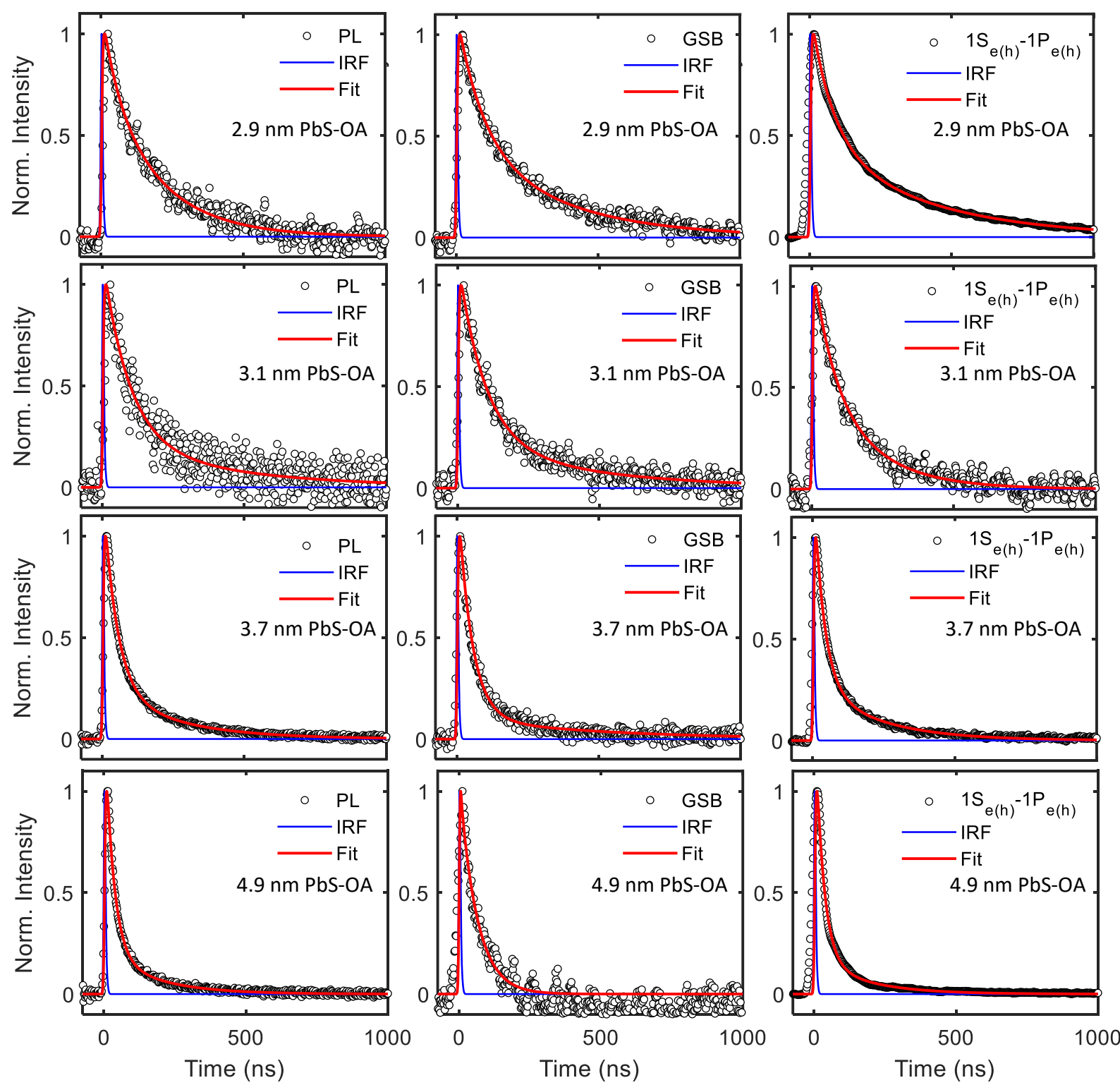

Figure S5. Transient absorption kinetics and PL decay traces for OA-PbS QD films with corresponding best fit biexponential functions. The parameters from the fitting procedure appear in Table $\mathbf{S 1}$. 
Biexponential decay functions were used to fit the decay curves using the form $S(t)=a^{*} \exp \left(-t / \tau_{1}\right)$ $+(1-a)^{*} \exp \left(-t / T_{2}\right)$, where $\left(\mathrm{T}_{1}\right)$ and $\left(\mathrm{T}_{2}\right)$ represent the short and long lifetimes respectively and (a) determines the contribution each makes to the overall decay function. All decay kinetics were convoluted with the instrument response function. The results of the fitting procedures and corresponding uncertainty limits determined the least squares fitting procedure are summarized in Table S1.
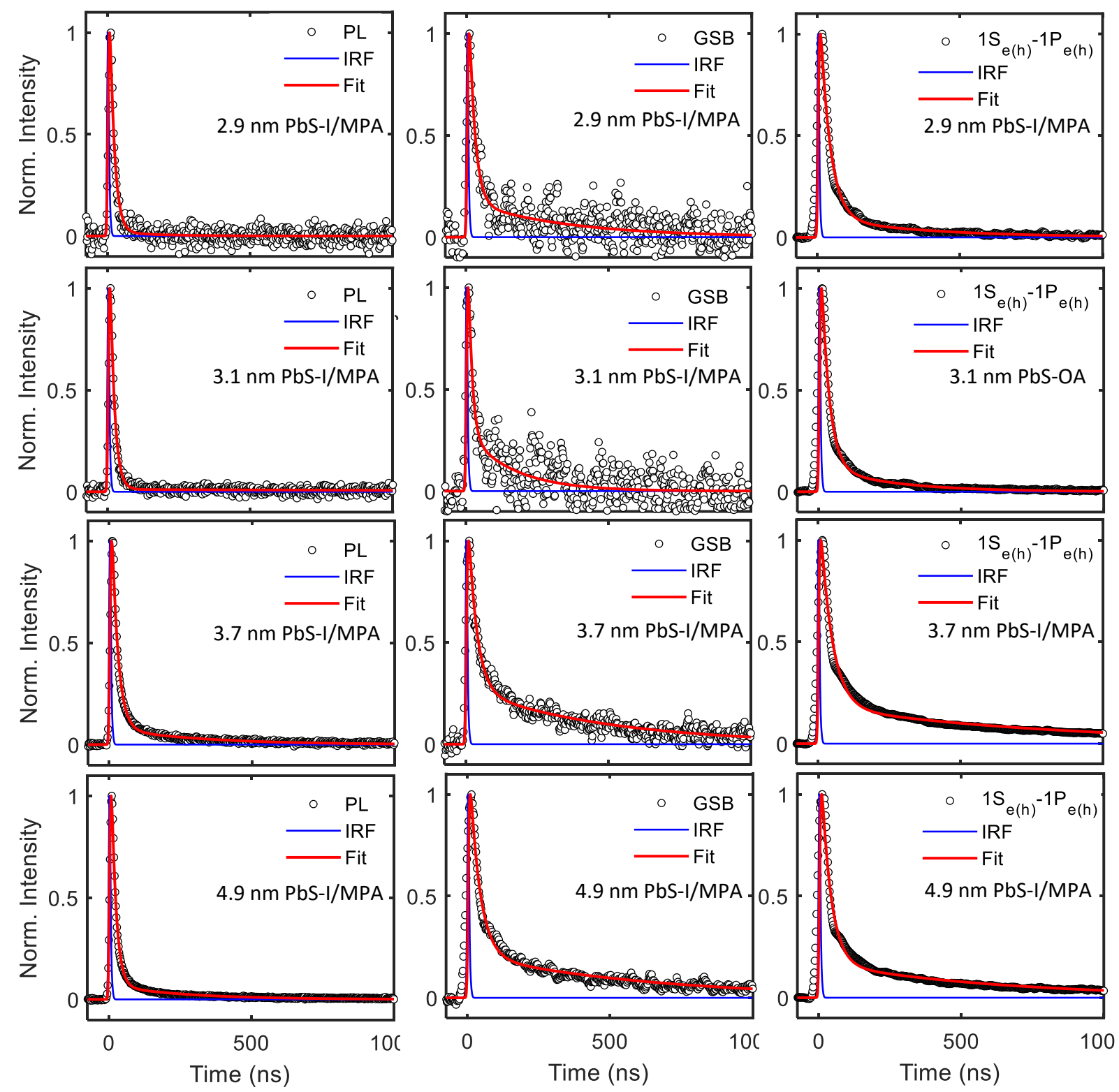

Figure S6. Transient absorption kinetics and PL decay traces for I-/MPA-PbS QD films with corresponding best fit biexponential functions. The parameters from the fitting procedure appear in Table S1. 
Table S1. Summary of best fit parameters and uncertainty limits from fitting transient absorption kinetics and PL decay traces appearing in Figure 4. Best fit curves are overlaid on the data in Figure S5 and Figure S6.

PbS-OA

\begin{tabular}{cccccc} 
Sample & Decay & $\mathbf{a}$ & $\mathbf{t}_{\mathbf{1}}$ (ns) & $\mathbf{t}_{\mathbf{2}}$ (ns) & $\mathbf{t}_{\text {ave }}$ (ns) \\
\hline $2.9 \mathrm{~nm} \mathrm{PbS} \mathrm{OA}$ & $\mathrm{PL}$ & $0.5 \pm .1$ & $80 \pm 10$ & $300 \pm 20$ & $190 \pm 50$ \\
& GSB & $0.52 \pm .05$ & $84 \pm 9$ & $330 \pm 20$ & $200 \pm 30$ \\
& $\mathrm{~S}-\mathrm{P}$ & $0.56 \pm .05$ & $78 \pm 9$ & $390 \pm 30$ & $220 \pm 40$ \\
\hline $3.1 \mathrm{~nm} \mathrm{PbS} \mathrm{OA}$ & $\mathrm{PL}$ & $0.79 \pm .05$ & $92 \pm 10$ & $430 \pm 80$ & $160 \pm 40$ \\
& GSB & $0.79 \pm .02$ & $94 \pm 6$ & $450 \pm 50$ & $170 \pm 20$ \\
& S-P & $0.79 \pm .04$ & $91 \pm 5$ & $400 \pm 20$ & $160 \pm 20$ \\
\hline $3.7 \mathrm{~nm} \mathrm{PbS} \mathrm{OA}$ & PL & $0.79 \pm .03$ & $48 \pm 2$ & $250 \pm 10$ & $90 \pm 6$ \\
& GSB & $0.79 \pm .04$ & $40 \pm 2$ & $230 \pm 10$ & $80 \pm 7$ \\
\hline $4.9 \mathrm{~nm} \mathrm{PbS} \mathrm{OA}$ & S-P & $0.77 \pm .03$ & $36 \pm 2$ & $220 \pm 30$ & $80 \pm 10$ \\
& PL & $0.83 \pm .02$ & $30 \pm 1$ & $170 \pm 10$ & $53 \pm 4$ \\
& GSB & $0.86 \pm .01$ & $40 \pm 5$ & $200 \pm 20$ & $62 \pm 12$ \\
& S-P & $0.86 \pm .01$ & $28 \pm 4$ & $170 \pm 20$ & $48 \pm 8$ \\
\hline
\end{tabular}

\section{PbS-I/MPA}

\begin{tabular}{cccccc} 
Sample & Decay & $\mathbf{a}$ & $\mathbf{t}_{\mathbf{1}}$ (ns) & $\mathbf{t}_{\mathbf{2}}$ (ns) & $\mathbf{t}_{\text {ave }}$ (ns) \\
\hline $2.9 \mathrm{~nm} \mathrm{PbS} \mathrm{I/MPA}$ & PL & $0.98 \pm .02$ & $16 \pm 1$ & $160 \pm 50$ & $18 \pm 6$ \\
& GSB & $0.88 \pm .05$ & $20 \pm 4$ & $180 \pm 50$ & $40 \pm 14$ \\
& S-P & $0.91 \pm .03$ & $27 \pm 4$ & $250 \pm 60$ & $48 \pm 14$ \\
\hline $3.1 \mathrm{~nm} \mathrm{PbS} \mathrm{I/MPA}$ & PL & $0.98 \pm .01$ & $15 \pm 1$ & $200 \pm 50$ & $18 \pm 5$ \\
& GSB & $0.81 \pm .05$ & $13 \pm 4$ & $160 \pm 50$ & $41 \pm 18$ \\
& S-P & $0.90 \pm .03$ & $28 \pm 4$ & $249 \pm 60$ & $48 \pm 14$ \\
\hline $3.7 \mathrm{~nm} \mathrm{PbS} \mathrm{I/MPA}$ & PL & $0.94 \pm .01$ & $21 \pm 1$ & $312 \pm 40$ & $37 \pm 5$ \\
& GSB & $0.79 \pm .02$ & $38 \pm 5$ & $530 \pm 30$ & $140 \pm 20$ \\
& S-P & $0.85 \pm .01$ & $42 \pm 2$ & $830 \pm 50$ & $160 \pm 10$ \\
& PL & $0.95 \pm .01$ & $16 \pm 1$ & $260 \pm 20$ & $27 \pm 3$ \\
& GSB & $0.86 \pm .01$ & $34 \pm 2$ & $640 \pm 60$ & $120 \pm 13$
\end{tabular}




\section{Section S4: References}

1. Grieco, C.; Doucette, G. S.; Pensack, R. D.; Payne, M. M.; Rimshaw, A.; Scholes, G. D.; Anthony, J. E.; Asbury, J. B., Dynamic Exchange During Triplet Transport in Nanocrystalline TipsPentacene Films. Journal of the American Chemical Society 2016, 138, 16069-16080.

2. Kang, I.; Wise, F. W., Electronic Structure and Optical Properties of Pbs and Pbse Quantum Dots. J. Opt. Soc. Am. B 1997, 14, 1632-1646.

3. Efros, A. L., Interband Light Absorption in Semiconductor Spheres. Sov. Phys. Semicond. 1982, 16, 772-775.

4. Deacon, G. B.; Phillips, R. J., Relationships between the Carbon-Oxygen Stretching Frequencies of Carboxylato Complexes and the Type of Carboxylate Coordination. Coord. Chem. Rev. 1980, 33, 227-250.

5. Kennehan, E. R.; Munson, K. T.; Doucette, G. S.; Marshall, A. R.; Beard, M. C.; Asbury, J. B., Dynamic Ligand Surface Chemistry of Excited Pbs Quantum Dots. J. Phys. Chem. Lett. 2020, 11, 2291-2297.

6. $\quad$ Miller, B. J.; Howard, D. L.; Lane, J. R.; Kjaergaard, H. G.; Dunn, M. E.; Vaida, V., ShStretching Vibrational Spectra of Ethanethiol and Tert-Butylthiol. J. Phys. Chem. A 2009, 113, 7576-7583. 